\title{
A comparative study of overlap and staggered fermions in QCD*
}

\author{
S. Dürra ${ }^{\mathrm{a}}$, Ch. Hoelbling ${ }^{\mathrm{b} \dagger}, \mathrm{U}$. Wenger ${ }^{\mathrm{c}}$ \\ ${ }^{a}$ DESY Zeuthen, Platanenallee 6, D-15738 Zeuthen, Germany \\ ${ }^{\mathrm{b}}$ Centre de Physique Théorique ${ }^{\ddagger}$, Case 907, CNRS Luminy, F-13288 Marseille Cedex 9, France \\ ${ }^{\mathrm{c}}$ NIC/DESY Zeuthen, Platanenallee 6, D-15738 Zeuthen, Germany
}

We perform a comparative study of the infrared properties of overlap and staggered fermions in QCD. We observe that the infrared spectrum of the APE/HYP improved staggered Dirac operator develops a four-fold near-degeneracy and is in quantitative agreement with the infrared spectrum of the overlap operator. The neardegeneracy allows us to identify the zero modes of the staggered operator and we find that the number of zero modes is in line with the topological index of the overlap operator.

\section{INTRODUCTION}

In the continuum the eigenmodes of the massless Dirac operator $D$ are described by the eigensystem $D \psi_{\lambda}=i \lambda \psi_{\lambda}, \lambda \in \mathbb{R}$. Since the operator enjoys chiral symmetry, $\left\{D, \gamma_{5}\right\}=0$, and is $\gamma_{5}$-hermitian, its spectrum is guaranteed to be symmetric around zero. The zero modes have a definite chirality, $\psi_{\lambda=0}^{\dagger} \gamma_{5} \psi_{\lambda=0}= \pm 1$, and are related to the topological charge of the background gauge field via the Atiyah-Singer index theorem which provides an explicit link to the axial $U_{A}(1)$ anomaly and hence guarantees that the fermions are sensitive to topology. On the other hand, the low-lying non-zero modes of the Dirac operator describe the low-energy, long-distance physics and are therefore referred to as the infrared (IR) modes.

A naive discretisation of the fermionic Dirac operator describes 16 flavours of fermions instead of one. A 4-fold degeneracy can be removed by distributing the spin degrees of freedom over a hypercube yielding the staggered Dirac operator

$D_{x, y}^{\mathrm{st}}=\frac{1}{2} \sum_{\mu} \eta_{\mu}(x)\left(U_{\mu}(x) \delta_{x+\hat{\mu}, y}-U_{\mu}^{\dagger}(x-\hat{\mu}) \delta_{x-\hat{\mu}, y}\right)$

\footnotetext{
*Talk presented by U.W.

†Supported by EU grant HPMF-CT-2001-01468.

¥Unité Mixte de Recherche (UMR 6207) du CNRS et des

Universités Aix Marseille 1, Aix Marseille 2 et sud ToulonVar, affiliée à la FRUMAM.
}

where $\eta_{\mu}(x)=(-1)^{\sum_{\nu<\mu} x_{\nu}}$. This operator enjoys a remnant $U(1)$ chiral symmetry which protects the bare quark mass from additive renormalisation, but it still describes 4 degenerate flavours in the continuum. These doubler fermions are light and mix with each other generating a complicated particle spectrum on the lattice. Moreover, since the operator generically has no zero modes, there is no (exact) index theorem and it is not clear, a priori, whether the operator is sensitive to topology.

There are other conceptual issues: in order to generate one flavour of sea quarks in a dynamical simulation, one usually employs the so-called fourth-root trick, i.e., one weights configurations by $\operatorname{det}\left(D^{\text {st }}\right)^{1 / 4}$ instead of $\operatorname{det}\left(D^{\text {st }}\right)$ which, however, is hard to justify from a field theoretical point of view. Even if it turns out that this is indeed a valid procedure, the question remains how one should match this prescription in the valence quark sector. The crucial point in this context is therefore whether the above discretisation of fermions provides a consistent field theoretic framework or whether it is just a model of QCD, albeit a good one.

One way of avoiding (at least some of) the doubling problem is to suppress the flavour changing interactions, i.e., to reduce the mixing of the doubler modes. This can be achieved by removing ultraviolet (UV) noise from the gauge fields by 
smearing the gauge links in one way or another. In the following we refer to this procedure as UV filtering or UV improvement and here we only note that any such procedure simply corresponds to a $O\left(a^{2}\right)$ redefinition of the staggered fermion action.

Another way is to make the doublers heavy, so that they decouple from the theory in the continuum limit. A particular realisation of this idea is provided by the overlap operator [1]

$$
D_{\mathrm{ov}}=\rho\left(1+\gamma_{5} \operatorname{sign}\left(\gamma_{5} D_{\mathrm{w}}(-\rho)\right)\right)
$$

where $D_{\mathrm{w}}$ is the Wilson Dirac operator. The advantages of this operator are manifold: there exists an exact lattice version of the continuum chiral symmetry which guarantees the absence of an additive renormalisation of the quark mass and avoids mixing of operators in different chiral representations. Furthermore, the operator possesses exact zero modes and allows to define an index theorem on the lattice implying the correct sensitivity of the operator to topology.

Here we report on our results from a comparative study of the spectra of the (UV improved) staggered and overlap Dirac operator [2]. We concentrate on the IR spectra of the staggered operator and compare it to the corresponding ones of the overlap operator as a benchmark. The question we would like to answer is whether one can see a 'continuum-like' behaviour in the spectra of the staggered operator similar to what has been observed in the Schwinger model [3], i.e. a four-fold near-degeneracy and approximate zero modes. Then we would like to know at what lattice spacing it appears, if at all, and whether any deviations scale like $O\left(a^{2}\right)$-artefacts. The final goal, however, is to eventually test whether

$$
\operatorname{det}\left(D_{\mathrm{st}}\right)^{1 / 4} \propto \operatorname{det}\left(D_{\mathrm{ov}}\right)+O\left(a^{2}\right) .
$$

\section{RESULTS}

We study the infrared spectra on four ensembles of quenched lattices with lattice spacings ranging from 0.18 to $0.07 \mathrm{fm}$ matched to have a fixed physical volume $V=(1.12 \mathrm{fm})^{4}$. We use standard smearing techniques and we note that

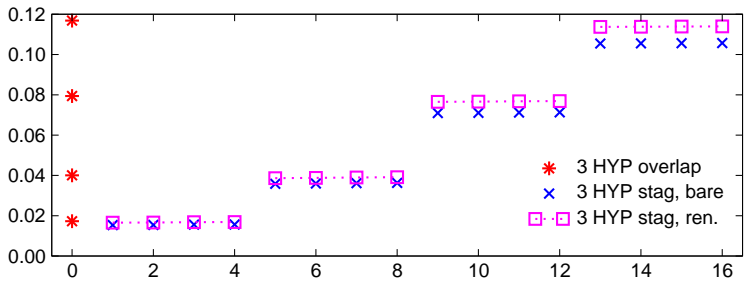

Figure 1. Overlap and staggered eigenvalues for one of our quenched configurations at $a \simeq 0.07 \mathrm{fm}$.

for the overlap operator we use $\rho=1.0$ throughout. We find that the overlap spectra are fairly stable under smearing for $a<0.1 \mathrm{fm}$. This indicates that the overlap operator separates well IR modes from UV fluctuations at small enough lattice spacings. The staggered spectra, on the other hand, change dramatically under repeated smearings. In particular, we find that without UV filtering there is no resemblance at all between the staggered and the overlap spectra. With increased UV filtering the staggered spectrum at large enough $\beta$ develops an (approximate) fourfold degeneracy and zero modes start to separate out. This suggests that an (approximate) index theorem holds for UV filtered staggered fermions. Moreover we find a one-to-one correspondence between the quadruples of staggered eigenvalues and the ones from the overlap operator. Occasionally, we find a mismatch of topological charge between the index of the overlap and the staggered Dirac operator. In particular we observe that whenever the overlap operator has difficulties to decide what charge to assign to a configuration, the staggered operator will have problems to produce a corresponding zero mode - instead, the four-fold degeneracy of the wouldbe zero mode remains rather approximate. We assign this behaviour to the occurrence of dislocations with a size of $O(1)$ lattice spacings which sometimes are detected as topological charges and sometimes not. It is clear, however, that even the overlap operator suffers from this ambiguity (generically, this also happens when changing the parameter $\rho$ in eq.(10) which suggests that this is a genuine $O\left(a^{2}\right)$ effect.

In figure \we plot the chirally rotated overlap eigenvalues most-left and those of the staggered 


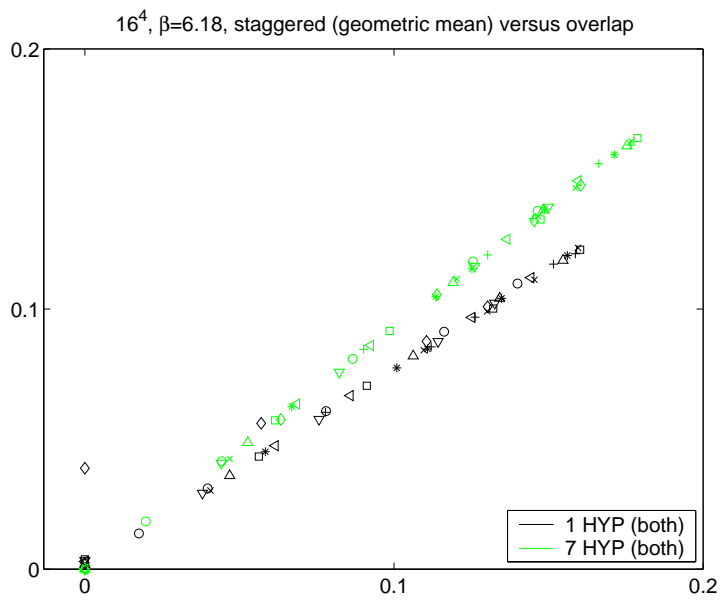

Figure 2. Staggered eigenvalues (geometric mean) vs. overlap eigenvalues at $a \simeq 0.07 \mathrm{fm}$. With only 1 HYP filtering, the overlap and staggered topological charges disagree on one configuration, with 7 HYP steps there is no such mismatch.

operator ordered according to increasing size for a generic configuration with $a=0.07 \mathrm{fm}$ and UV filtering level HYP3 2. After rescaling the staggered spectra with the same factor for all configurations, they show a quantitative agreement with the corresponding spectra of the overlap operator (up to mismatches in the topological charge as discussed above).

In figure 2 we plot the geometric mean of the staggered eigenvalues against the eigenvalues of the corresponding overlap operator for filtering levels HYP1 and HYP7 on our quenched ensemble at $a \simeq 0.07 \mathrm{fm}$. It is evident that there is a linear correspondence between the UV filtered overlap eigenvalues and the staggered eigenvalue quadruples at small accessible couplings and with only moderate UV filtering. As emphasised before, the correspondence of the (would-be) zero modes suggests an index theorem for improved staggered fermions up to $O\left(a^{2}\right)$ artefacts. The one-to-one correspondence also implies that improved staggered fermions show the same sensitivity to topology as overlap fermions and fall into the same universality class of random matrix theory [4. Indeed, this has recently been verified [56].

\section{CONCLUSIONS AND OUTLOOK}

We have found a four-fold degeneracy in the IR spectra of improved staggered Dirac operators up to $O\left(a^{2}\right)$ artefacts, hence providing evidence that the rooting-procedure of the staggered determinant might be justified in the sense of eq. (2). We found a quantitative correspondence between the IR part of the improved staggered and overlap spectra in QCD on individual configurations and our data are compatible with the assumption that the remaining artefacts scale as $O\left(a^{2}\right)$ with moderate UV filtering and at accessible couplings. However, a number of conceptual issues within the staggered fermion formulation of QCD remain. In particular it is not clear, whether and how a local operator $D$ with $\operatorname{det}(D)=\operatorname{det}\left(D_{\text {stag }}\right)^{1 / 4}$ can be constructed with the staggered degrees of freedom.

Finally, we note that our results could point the way towards unquenching the overlap Dirac operator via a 'hybrid HMC' algorithm: one could use an improved staggered Dirac operator along the molecular dynamics trajectory and accept/reject the final configuration at the end of the trajectory with an overlap Dirac operator. The hope is that in this way a correct dynamical overlap ensemble could be generated essentially at the cost of a dynamical staggered fermion simulation.

\section{REFERENCES}

1. R. Narayanan and H. Neuberger, Nucl. Phys. B 412 (1994) 574 arXiv:hep-lat/9307006.

2. S. Dürr, C. Hoelbling and U. Wenger, arXiv:hep-lat/0406027.

3. S. Dürr and C. Hoelbling, Phys. Rev. D 69 (2004) 034503 arXiv:hep-lat/0311002,

4. E. V. Shuryak and J. J. M. Verbaarschot, Nucl. Phys. A 560, 306 (1993) arXiv:hep-th/9212088.

5. E. Follana, A. Hart and C. T. H. Davies [HPQCD Collaboration], arXiv:hep-lat/0406010 E. Follana, arXiv:hep-lat/0409062.

6. K. Y. Wong and R. M. Woloshyn, arXiv:hep-lat/0407003 